\title{
ON THE RELATIVISTIC CALCULATION OF THE LAMB SHIFT
}

\author{
ROGER BOUDET \\ Université de Provence \\ Pl. V. Hugo, F-1331 Marseille, France
}

\begin{abstract}
A relativistic calculation of the Lamb shift, using the classical field created by the Dirac transition currents, is proposed.

1. Introduction. The question concerning the respective validities of the use of the quantum field theory (QFT) and the semi-classical theory (SCT), for the calculation of the Lamb shift, ought to be definitively closed.

Though the construction of the formulas used in both cases are conceptually different, in the last resort, these formulas are exactly the same: the use in QFT of the threedimensional perturbation theory ([1], p. 364) together with the abandon of the dipole approximation, give the same formulas as the SCT coupling of the unquantified Maxwell laws for the self-field, with the quantum equations (Schrödinger or Dirac) of the electron [2], [3].

The fact that the dipole approximation is the only cause of the QFT divergences is now accepted by the QFT authors [4]-[7], and therefore the term of "Finite Quantum Electrodynamics" introduced by A.O. Barut for the SCT calculation is quite convenient for the QFT one. However it is impossible not to concede the virtue of conceptual simplicity to the pure Maxwell laws, in regard with the complicated creation-annihilation operators theory.
\end{abstract}

The present paper aims:

a) on one side, to give some justifications about the relativistic classical electromagnetic formulas used in the SCT calculation of the shift, in particular those relative to their links with other quantum phenomenas in which the use of the classical field appears as a necessity,

b) on the other, to establish the general analytic expression of the shifts of the $\mathrm{S} 1 / 2$, P1/2, P3/2 states.

1991 Mathematics Subject Classification: Primary 83A05; Secondary 81Q05.

The paper is in final form and no version of it will be published elsewhere. 
The way followed here is that of the finite electrodynamics model [8] in which the field is classical and the nature, quantal or classical of the source current of the field does not play an essential role, only the mathematical properties of the current beeing taken into account.

Therefore the calculation of the shift lies on the calculation of the interaction energy of the time-periodic Dirac transition currents between states in a hydrogenic atom [9], these same currents which bring an outstanding confirmation of the validity of the use of the classical field created by an quantum electron in the explanation of the anomalous Zeeman effect, as well for the polarization of light in this effect as for the prediction of the ratios of the intensities of the Zeeman components.

2. The Lamb shift standard formula. Let us consider some general system $S$ of charges which can have stationary states, each corresponding to some constant energy level $E_{n}$. We suppose that the transition current between two states $n$ and $p$ is timeperiodic:

$$
\mathbf{j}_{n p}\left(x^{0}, \mathbf{r}\right)=e\left(\cos \Omega_{n p} x^{0} \mathbf{j}_{1 n p}(\mathbf{r})+\sin \Omega_{n p} x^{0} \mathbf{j}_{2 n p}(\mathbf{r})\right),
$$

where $e$ is the total charge of the system.

The self-energy associated with $E_{n}$ in the transition $(n, p)$ is deduced from the timeaveraged interaction energy of the transition and is given in the Coulomb gauge by (see $[10],[11])$

$$
\begin{gathered}
w_{n p}=w_{1 n p}+w_{2 n p} \\
w_{X n p}=\frac{\Omega_{n p} e^{2}}{16 \pi^{2}} \int \frac{\mathbf{T}_{X n p}^{\perp}(-\mathbf{k}) \cdot \mathbf{T}_{X n p}^{\perp}(\mathbf{k})}{\left(\Omega_{n p}-k\right)} \frac{d \mathbf{k}}{k^{2}} \\
\mathbf{T}_{X n p}^{\perp}(\mathbf{k})=\int e^{i(\mathbf{k} \cdot \mathbf{r})} \mathbf{j}_{X n p}^{\perp}(\mathbf{r}) d \mathbf{r}, \quad X=1,2
\end{gathered}
$$

in which the symbol $\mathbf{j}^{\perp}$ means the component of the vector $\mathbf{j}$ orthogonal to $\mathbf{k}$ :

$$
\mathbf{j}^{\perp}=\mathbf{j}-(\mathbf{j} . \mathbf{K}) \mathbf{K}, \quad \mathbf{K}=\mathbf{k} / k, \quad k=|\mathbf{k}| .
$$

Considering the self-energy associated with one state $n$, we take the sum $W_{n}$ of the self-energies $w_{n p}$ for all the transitions $(p, n)$ :

$$
W_{n}=\sum_{p} w_{n p}
$$

If one considers that the current $\mathbf{j}_{n p}\left(x^{0}, \mathbf{r}\right)$ is the Schrödinger transition current between the two states $n$ and $p$ of an electron in an hydrogenic atom, if one denotes $\Omega_{n p}=\left(E_{n}-E_{p}\right) / \hbar c$, then the above formula is exactly the same as the non relativistic Lamb shift formula established by Barut and Van Huele [3] by coupling the Schrödinger equation with the Maxwell equation of the self-field.

Two important questions arise now.

a) The formula has been settled in the Coulomb gauge by using the half retardedadvanced potential. Is it coherent with phenomenas as the Zeeman effect or the spontaneous emission, in wich only the retarded potential is to be considered? 
b) Is it possible to use this formula in a relativistic way by replacing the Schrödinger currents by the Dirac currents?

The answer to the second question lies in the answer to the first one.

a) We have proved in [11] that, if one uses the Lorentz gauge, the time-averaged interaction energy of a time-periodic current is independant of the nature, retarded, advanced or half retarded-advanced, of the potential. One can prove without difficulty the following theorem:

If the charge current is time-periodic and decreases sufficiently rapidly at infinity, its time-averaged interaction energy is gauge independant.

Because of the presence of a factor $\exp (-\lambda r)$ in the transition current from a state of the discrete or the continuous spectrum, to a state of the discrete spectrum, the gauge independance (and so the compatibity of the formula with the choice of the retarded potential) is therefore carried out in the formula of the shift of a level of the discrete spectrum.

b) As the Lorentz gauge is relativistic, the current which is used may be the Dirac current.

We emphasize that there is no theoritical reason to use one gauge instead of the other. In QFT the elimination of the "longitudinal photons" is a necessity, and the classical analogue of such an operation is the use of the Coulomb gauge. But in classical theory of the field there is no distinction to make between "transverse" or "longitudinal" photons, because there is no photon at all to consider. In reality the only reason to prefer the Coulomb gauge is the fact that its use is much more simpler than Lorentz's one because the Coulomb gauge eliminates the scalar potential.

\section{The analytic formulas of the Lamb shift.}

3.1. The Dirac transition currents. We have established in [9] the general expression of the Dirac transition currents for the Darwin solutions of the central potential problem.

Let $\left(\mathbf{e}_{\mathbf{1}}, \mathbf{e}_{\mathbf{2}}, \mathbf{e}_{\mathbf{3}}\right)$ be an orthonormal frame of the $E^{3}$ space and let us denote

$$
\begin{gathered}
\mathbf{u}=\cos \varphi \mathbf{e}_{1}+\sin \varphi \mathbf{e}_{2}, \quad \mathbf{v}=-\sin \varphi \mathbf{e}_{1}+\cos \varphi \mathbf{e}_{2}, \\
\mathbf{n}=\cos \theta \mathbf{e}_{3}+\sin \theta \mathbf{u}, \quad \mathbf{r}=r \mathbf{n} .
\end{gathered}
$$

For $\Delta m=0(m$ : azimuthal quantum number $)$ the transition current between two states $\psi_{1}$ and $\psi_{2}$ is of the form

$$
\mathbf{j}_{1}(\mathbf{r})=B(r, \theta) \mathbf{v}, \quad \mathbf{j}_{2}(\mathbf{r})=A(r, \theta) \mathbf{u}+C(r, \theta) \mathbf{e}_{3}
$$

with in particular

$\psi_{1}=S 1 / 2, \psi_{2}=P 1 / 2$ :

$$
\begin{gathered}
B=0, \quad A=\frac{1}{2 \pi} 2 g_{2} f_{1} \cos \theta \sin \theta, \quad C=\frac{1}{2 \pi}\left(g_{2} f_{1}\left(2 \cos ^{2} \theta-1\right)-g_{1} f_{2}\right) \\
\psi_{1}=S 1 / 2, \psi_{2}=P 3 / 2: \\
B=\frac{3}{2 \pi \sqrt{2}}\left(g_{2} f_{1}+g_{1} f_{2}\right) \cos \theta \sin \theta, \quad A=\frac{1}{2 \pi \sqrt{2}}\left(3 g_{1} f_{2}-g_{2} f_{1}\right) \cos \theta \sin \theta
\end{gathered}
$$




$$
C=\frac{1}{2 \pi \sqrt{2}}\left[g_{1} f_{2}\left(3 \cos ^{2} \theta-1\right)-g_{2} f_{1}\left(3 \cos ^{2} \theta+1\right)\right]
$$

in which $g(r)$ and $f(r)$ means the great and fine radial functions.

3.2. Calculation of the vectors $\mathbf{T}^{\perp}( \pm \mathbf{k})$. Denoting

$$
\begin{gathered}
\mathbf{K}=\cos \theta_{0} \mathbf{e}_{3}-\sin \theta_{0} \mathbf{e}_{1}^{\prime}, \quad \mathbf{e}_{1}^{\prime}=\cos \varphi_{0} \mathbf{e}_{1}+\sin \varphi_{0} \mathbf{e}_{2} \\
\mathbf{I}=\cos \theta_{0} \mathbf{e}_{1}^{\prime}+\sin \theta_{0} \mathbf{e}_{3}, \quad \mathbf{J}=\mathbf{K} \times \mathbf{I}, \quad \mathbf{n}=\cos \hat{\theta} \mathbf{K}+\sin \hat{\theta}(\cos \hat{\varphi} \mathbf{I}+\sin \hat{\varphi} \mathbf{J})
\end{gathered}
$$

in such a way that $\mathbf{e}_{3}^{\perp}=\sin \theta_{0} \mathbf{I}, \quad \mathbf{k} . \mathbf{r}=k r \cos \hat{\theta}$.

Using the relations in the spherical triangles $\left(\mathbf{n}, \mathbf{K}, \mathbf{e}_{3}\right)$ and $\left(\mathbf{n}, \mathbf{K}, \mathbf{e}_{1}^{\prime}\right)$

$$
\begin{gathered}
\text { n.e } \mathbf{e}_{3}=\cos \theta=\cos \hat{\theta} \cos \theta_{0}+\sin \hat{\theta} \sin \theta_{0} \cos \hat{\varphi} \\
\text { n.e } \mathbf{e}_{1}^{\prime}=\sin \theta \cos \left(\varphi-\varphi_{0}\right)=-\cos \hat{\theta} \sin \theta_{0}+\sin \hat{\theta} \cos \theta_{0} \cos \hat{\varphi}
\end{gathered}
$$

and, in the $\left(\theta, \varphi^{\prime}\right)$ and the $(\hat{\theta}, \hat{\varphi})$ spherical coordinates of $\mathbf{n}$,

$$
\begin{gathered}
\int_{-\pi}^{\pi} e^{ \pm i(\mathbf{k} . \mathbf{r})} \sin \varphi^{\prime} d \varphi^{\prime}=0 \quad\left(\varphi^{\prime}=\varphi-\varphi_{0}\right) \\
\int_{0}^{\pi} e^{ \pm i(\mathbf{k} . \mathbf{r})} \sin \hat{\theta} d \hat{\theta}=2 j_{0}(k r), \quad \int_{0}^{\pi} e^{ \pm i(\mathbf{k} . \mathbf{r})}\left(1-3 \cos ^{2} \hat{\theta}\right) \sin \hat{\theta} d \hat{\theta}=4 j_{2}(k r)
\end{gathered}
$$

in which $j_{0}, j_{2}$ are the spherical Bessel functions

$$
j_{0}(\rho)=\frac{\sin \rho}{\rho}, \quad j_{2}(\rho)=\left(-\frac{1}{\rho}+\frac{3}{\rho^{3}}\right) \sin \rho-\frac{3}{\rho^{2}} \cos \rho .
$$

Denoting

$$
L_{s, i j}=\int_{0}^{\infty} j_{s}(k r) g_{i}(r) f_{j}(r) r^{2} d r
$$

one finds without difficulty

$$
\psi_{1}=S 1 / 2, \psi_{2}=P 1 / 2 \text { : }
$$

$$
\begin{aligned}
\mathbf{T}_{1}^{\perp}( \pm \mathbf{k})=0, \quad \mathbf{T}_{2}^{\perp}( \pm \mathbf{k})=\sin \theta_{0}\left[\frac{4}{3} L_{2,21}(k)-\frac{2}{3} L_{0,21}(k)-2 L_{0,12}(k)\right] \mathbf{I} \\
\psi_{1}=S 1 / 2, \psi_{2}=P 3 / 2:
\end{aligned}
$$

$$
\mathbf{T}_{1}^{\perp}( \pm \mathbf{k})=0, \quad \mathbf{T}_{2}^{\perp}( \pm \mathbf{k})=\sqrt{2} \sin \theta_{0}\left[L_{2,12}(k)-\frac{1}{3} L_{2,21}(k)-\frac{4}{3} L_{0,21}(k)\right] \mathbf{I} .
$$

3.3. Calculation of the contribution of a state to the shift of a level. Using the $\left(\theta_{0}, \varphi_{0}+\right.$ $\pi$ ) spherical coordinates of $\mathbf{K}$, because

$$
\frac{d \mathbf{k}}{k^{2}}=\sin \theta_{0} d \varphi_{0} d \theta_{0} d k, \quad \int_{0}^{2 \pi} \int_{0}^{\pi}\left(\sin ^{2} \theta_{0}\right) \sin \theta_{0} d \varphi_{0} d \theta_{0}=\frac{8 \pi}{3}
$$

one obtains in the above examples for the contribution of a $P$ state of energy $E_{2}$ to the shift of the level of energy $E_{1}$ of a $S$ state in the hydrogen atom

$$
\Delta E_{12}=\frac{\alpha}{6 \pi}\left(E_{1}-E_{2}\right) \int_{0}^{\infty} \frac{M_{12}(k)}{\frac{E_{1}-E_{2}}{\hbar c}-k} d k, \alpha=e^{2} / \hbar c
$$

(the presence of the factor $1 / 6$ instead to the expected factor $2 / 3$ is due to a strict definition of the transition currents, see [5], p. 1390 and [8], eq. 17) with 


$$
\psi_{1}=S 1 / 2, \psi_{2}=P 1 / 2:
$$

$$
M_{12}(k)=\left[\frac{4}{3} L_{2,21}(k)-\frac{2}{3} L_{0,21}(k)-2 L_{0,12}(k)\right]^{2}
$$

$\psi_{1}=S 1 / 2, \psi_{2}=P 3 / 2:$

$$
M_{12}(k)=2\left[L_{2,12}(k)-\frac{1}{3} L_{2,21}(k)-\frac{4}{3} L_{0,21}(k)\right]^{2} .
$$

As an example one obtains in the approximation $\alpha^{2}<<1$ ( $a=$ Bohr radius) $\psi_{1}=S 1 / 2, \psi_{2}=P 1 / 2$ :

$$
\Delta E^{(1)} / \hbar c=\frac{\alpha^{4}}{a \pi} \frac{2^{7}}{3^{8}} \frac{1}{3}\left[\ln \frac{4}{\alpha}-\frac{137}{120}+\frac{6}{5}+\frac{\alpha \pi}{8}\right] \text { or } \nu=33.7 \mathrm{MHz}
$$

$\psi_{1}=S 1 / 2, \psi_{2}=P 3 / 2$ :

$$
\Delta E^{(2)} / \hbar c=\frac{\alpha^{4}}{a \pi} \frac{2^{7}}{3^{8}} \frac{2}{3}\left[\ln \frac{4}{\alpha}-\frac{137}{120}-\frac{3}{32}+\frac{\alpha \pi}{8}\right] \text { or } \nu=53.7 \mathrm{MHz}
$$

and so a total contribution of the $2 P$ states to the $1 S 1 / 2$ level (with $\Delta m=0$ ) of $\nu=87.37$ $\mathrm{MHz}$.

This value is to be compared to the one obtained in [10] in the non relativistic calculation for the contribution of $2 p_{0}$ to the shift of $1 s$ :

$$
\Delta E / \hbar c=\frac{\alpha^{4}}{a \pi} \frac{2^{7}}{3^{8}}\left[\ln \frac{4}{\alpha}-\frac{11}{12}+\frac{\alpha \pi}{8}\right] \text { or } \nu=85.6 \mathrm{MHz}
$$

i.e. a difference of about 2 per cent.

4. Conclusion. The analytic expression one can obtain for the Lamb shift is not simple because of the complexity of the radial functions, and the fact that, after the calculation of each of the convergents integrals expressing the contribution of a state to the schift of a given level, one has to carry out the calculation a) of a series for the contribution of the discrete spectrum, b) an integral for the contribution of the continuum (nothing above gives the assurance that this series and this integral converge). However it seems that the direct calculation is the only way to avoid the approximations which are made in the calculation of the non-relativistic Bethe formula which is still used, to my knolewdge, for the low-energy part of the calculation of the shift.

Such a direct calculation, carried up to all wanted degree of precision, does not appear as beeing beyond the present possibilities given by the use of a computer. As a beginning, it is a simple matter to extend to $1 S 1 / 2$, with the approximation $\alpha^{2}<<1$, the calculation of the contribution of the discrete spectrum to the shift of $1 s$ which has been made in [12] (in which one can see that the series converge very quickly) with the help of the Schrödinger transition currents . Though the QFT numerical results of the shifts which are presently obtained, in particular in calculations "with retardation", are in excellent agreement with experiments, a confirmation by the other way of the direct relativistic calculation seems desirable. 


\section{References}

[1] H. Grotch, D.R. Yennie, Rev. Mod. Phys., 41, 350 (1969).

[2] A.O. Barut, J. Kraus, Found. Phys, 13, 189 (1983).

[3] A.O. Barut, J. Van Huele, Phys. Rev. A, 32, 3187 (1985).

[4] N.M. Kroll, in Quantum Optics and Electronics (Gordon and Breach, N. Y., 1965), pp. $51-53$.

[5] H.S. Hoffman, H.E. Moses, Lett. al Nuovo Cimento, 4, 54 (1972).

[6] C.K. Au, G. Feinberg, Phys. Rev. A, 9, 1794 (1974).

[7] H. Grotch, Am. J. Phys., 49, 48 (1988).

[8] R. Boudet, Found. Phys. Lett., 3, 311 (1990).

[9] R. Boudet, Found. Phys. 23, 1387 (1993).

[10] B. Blaive, R. Boudet, Ann. Fond. L. de Broglie, 14, 147 (1989).

[11] R. Boudet, Ann. Fond. L. de Broglie, 14, 119 (1989).

[12] B. Blaive, A.O. Barut, R. Boudet, J. Phys. B24, 3121 (1991). 